\title{
Clinical Problems of Motor System Disease
}

\author{
J. C. RICHARDSON
}

SUMMARY: This prologue to a symposium of research studies on motor mechanisms is a general commentary by a clinical neurologist. The vast extent and intricacy of modern basic neurological scientific knowledge presents a rather bewildering challenge to reasonable clinical application. In some degree this is being handled by complex and diverse neurological subspecialization. It is recalled that many past advances in the knowledge of neurological disease were achieved by a series of alternating and supporting bedside and laboratory observations and studies.

RÉSUMÉ: Ce prologue au symposium sur les mécanismes moteurs est un commentaire général apporté par un neurologue clinicien. Les nombreux dérangements dans le mouvement et le tonus musculaire qui précèdent le désordre des mécanismes moteurs vont continuer à rechercher des explications pour lesquelles le modèle humain sera toujours
The varied disorders of movement and muscle tone which signal disordered motor mechanisms will continue to demand explanation and will keep the human model in a leading research position. Clinical and laboratory research leading to part discoveries of mechanisms of disease is sometimes productive of dramatic new means of therapy. The story of Wilson's disease is briefly reviewed in that context. Some recent studies on hypoxic myoclonus are described with the evidence of a serotonin defect and useful related therapy.

utile. La recherche clinique et de laboratoire qui conduit à des découvertes partielles des mécanismes de la maladie est quelquefois productrice de nouvelles méthodes de thérapie. L'histoire de la maladie de Wilson est brièvement revue dans ce contexte. Quelques études récentes sur le myoclonus hypoxique sont également décrites.
From the section of neurology, Toronto General Hospital.

Reprint requests to Dr. J. C. Richardson, Ste. 305-170 St. George Street, Toronto, Ontario MSR $2 \mathrm{M8}$.
It is pleasing to be included as a speaker at this Symposium and it has been instructive and challenging to read and think about new and old studies on motor nervous mechanisms. However, unlike all the other papers by investigators who are speaking of their own recent research work, my contribution is that of a physician neurologist with no scientific pretensions. But it does seem justified to speak from the perspective of a very interested observer and an eager consumer of the products of the neurological science laboratories. Perhaps I may speak briefly of the complexities of modern neurology and then review some neurological discoveries achieved by varied efforts of the laboratory and the clinic.

Of the massive growth of new knowledge concerning the nervous system, a large share is about the motor apparatus. Each site of motor activity has been explored minutely by many techniques. The muscle spindle and the motor end plate have become revealed with amazing intricate knowledge of molecular structure, as well as electrical and chemical details of function. The lower motor neuron is now viewed and understood with a detail never imagined in speaking of the final common pathway a few decades ago. And the hierarchy of motor cortex, basal ganglia and cerebellum in their connections and functional interrelations, are being viewed in the light of new scientific scrutiny and interpreted with great promise. These modern in-depth studies of neuronal motor activities depend upon sophisticated new techniques and apparatus from various fields of medical research and general science. 
And needless to say the extent and excellence of the acquired and expanding neurobiological knowledge depend on subspecialization and extensive collaboration of research scientists in many fields.

The clinical neurologist dealing with motor symptoms in human disease, faces his task with a perspective changed greatly by all the new knowledge from the laboratory. Of necessity his viewpoint centers on the recognition and treatment of disease, whereas the fundamental researcher is mainly interested in discovering the minute structure and detailed mechanisms of the nervous system. Fortunately their perspectives and interests are not incompatible; clinicians are curious about how the brain works and the neuroscientist is not entirely uninterested in the cure of disease. Subspecialization has been inevitable in the clinic as well as the laboratory. Modern medical neurology of excellence depends on experts - paediatric neurologists, neuro-opthalmologists, aphasiologists, neuropharmacologists, neuroimmunologists, stroke doctors and not least, the neuromuscular experts. The physician scientist plays an important and a difficult role in the whole neurological production.

Briefly, to carry the banner for traditional clinical neurology it seems fair to say that studies of motor disorders produced by disease as well as by ablations and stimulation, require skill and precision. Such fundamental acumen in the examination of patients has been noticeably weakened by the many new tools of neurological diagnosis and research. Sir Gordon Holmes developed the technique of neurological examination to an unexcelled degree, a clinical science in itself. Tributes written before and since his death ten years ago (Walshe, 1956; Aring, 1965; Denny-Brown, 1956; Penfield, 1967), emphasize the tremendous influence of his teaching and writings. We who had the good fortune to work with him as clinical clerk and house physician have continued to practise and teach what he taught, forty years ago. One of Gordon
Holmes greatest works was with cerebellar disease and function. His description of cerebellar tests and signs, and his topical classification of cerebellar syndromes, retain their accuracy and acceptance even in the bright light of the amazing modern detailed knowledge of cerebellar circuitry and function (Holmes, 1939).

A survey of our present understanding of neurological disease calls for a broad look at past advances and how they were brought about. Viewed simply, the usual sequence is one of an original clinical identification of a new neurological disease or syndrome with resultant scientific curiosity and enlightenment by laboratory research. That in turn spurs the clinician to sharper and deeper clinical knowledge, sometimes with spectacular therapy. This pattern of alternating and overlapping progression of activity by clinician and researcher has some classical examples. Parkinson's description of the shaking palsy provoked pathological and physiological studies during the subsequent one and a half centuries, and the important biochemical and pharmacological discovers of recent years. Hughlings Jackson's meticulous observations of his patients and his brilliant prophetic deductions fostered a century of scientific laboratory and surgical studies of epilepsy. His conception of the discharging lesion, a "physiological fulminate" of nerve cells, still awaits full physicochemical interpretaion. The same clinical and laboratory pattern of accumulating knowledge can be recalled in many other diseases such as Huntington's chorea, myasthenia gravis and Wilson's disease.

The neurological signs of damaged motor mechanisms in human disease are abnormalities of movement and muscular tone, such as tremor, chorea, dysmetria, myoclonus, rigidity and dystonia. Some motor signs such as fasciculation, paralysis and incoordination have become clarified by prolonged collaborative efforts of neuroscientist and clinician. Others such as chorea and dystonia remain very incompletely understood. In all events these motor symptoms in both man and animal remain crucial indices of motor mechanisms. Not only are they diagnostic evidence of neurological disease, they are potential clues for further solution of the mysteries of neural motor function.

Our present understanding of such signals of deranged motor mechanisms has been gained from various sources. Much has depended on the human model. For some time knowledge of these signs, and related functions particularly of basal ganglia and cerebellum, depended entirely on clinical observation of diseased or injured patients, with anatomical and pathological correlation. More recently there has been an added source of information from neurosurgical ablations and stereotactic lesions. Still further the human model has been studied profitably by electrical stimultion of brain and spinal cord during neurosurgical operations. A newer study is directed to the drug induced movement disorders related to reserpine, phenothiazines and L-dopa. Pharmacological studies with increasing knowledge of neurotransmitters promise other important advance.

The animal research model of various species continues to provide abundant knowledge, particularly in regard to lower levels of motor function. Studies of higher central motor mechanisms in the monkey and ape have become increasingly sophisticated and productive. The ablation, stimulation and drug techniques are extended well beyond the feasibility in the human model, but with the limitations inherent with the subhuman nervous apparatus.

Aside from neurological research studying basic motor mechanisms and research into the primary cause and complete cure of disease, such as poliomyelitis, there is also research into limited manifestations of disease. Such part studies of disease may lead to dramatic therapeutic discoveries as well as intriguing challenges of further study. Two such examples now to be mentioned are Wilson's disease and hypoxic myoclonus. 
Wilson's disease, hepatolenticular degeneration, provided an outstanding example of research giving a part answer with brilliant therapeutic results and wide implications. This familial degenerative disease with a strange combination of basal ganglion and liver involvement was described by Kinnier Wilson in 1912 . The cerebral symptoms are predominantly motor, featured by severe tremor of mixed types. In 1948 it was found that a patient with Wilson's disease had a much increased urinary copper content. This was a fine example of serendipity in that the research workers were really screening cases of multiple sclerosis for a copper disturbance because of the evidence that swayback, a demyelinating disease of lambs, seemed to be based on a copper deficiency (Mandelbrote et al., 1948). It was a dramatic breakthrough in which a hopeless familial disease proved to be a biochemical disorder with copper poisoning of brain and liver. Of immediate practical importance was the finding that the disease could be arrested and the cerebral symptoms largely cleared by chelation therapy, best continued by penicillamine. The ten adult patients treated at the Toronto General Hospital since 1956 are all well controlled and nearly all of them, symptom free.

Continuing laboratory research on Wilson's disease in the past quarter century has struggled to detect the basic metabolic defect. The evidence for a faulty formation of the copper binding protein, caeruloplasmin has proved unconvincing. Recently interest has centred on the reduced copper excretion in bile. There is evidence of an hepatic lysosomal defect of copper excretion in an impressive study from Albert Einstein College (Sternlieb et al., 1973). Soon there will be full scientific understanding of what seemed to be a chronic degenerative familial disease.

Lance and Adams in 1963 described "The Syndrome of Intention or Action Myoclonus as a Sequel to Anoxic Encephalopathy". Their paper is a fine example of traditional expert clinical elicitation and analysis of the unique motor symptoms with added competent use of methods and knowledge of applied neurophysiology. Their four patients with brain hypoxia from respiratory or cardiac arrest presented a constant pattern of violent myoclonic jerking or synergistic and stabilizing trunk and limb muscles brought about by active purposive movements of the legs in standing and walking, and of the upper limbs in attempted actions of hands and arms. Further, they noted and studied the transient akinesia following the violent myoclonic jerks of trunk and proximal limb musculature.

In all cases there was an added feature of some cerebellar dysmetria and tremor. Their studies led them to conclude that the mechanisms of the myoclonus involved thalamocortical projections as well as brainstem and spinal cord levels of dysfunction. "It is suggested that the basic disturbance in intention or action myoclonus is the synchronous and repetitive firing of cells in the ventrolateral thalamic nucleus in response to afferent impulses from the cerebellum, and that the resulting thalamocortical volleys are relayed down the corticospinal tract as myoclonic impulses."

In the decade following the Lance and Adams paper, there have been reported about fifteen more patients with this syndrome of action myoclonus and cerebellar incoordination following varied cerebral anoxic accidents. Noteworthy are the reports by Parisian neurologists. The one necropsy study was by Castaigne, Cambier and Escourolle, (1964). Lesions were seen in the body of Luys, the ventrolateral and medial thalamic nuclei and slightly in the tegmentum. The dentate and red nuclei and superior cerebellar peduncle appeared unaffected. Unlike the usual anoxic encephalopathy, their case showed no lesions in the cerebral cortex or white matter. Minor changes were seen in the putamen and caudate nuclei. The cerebellum seemed unaffected save for a minor loss of Purkinje cells. This differs from Irving Cooper's case in which cerebellar biopsy showed marked.loss of Purkinje cells.

In the past year we have studied two new examples of this fascinating movement disorder at the Toronto General Hospital - 1 61-year-old workman with anoxic brain damage following chest injury, and a 75-year-old woman who emerged from coma following barbiturate poisoning, with the same condition. Like the other small group of reported cases they retained normal mentality and were free from cerebral cortical symptoms, but did show the striking pure combination of violent disabling coarse myoclonus brought out by intended movements and cerebellar ataxia. Dr. Jacques DeLean in consultation with Dr. O. Hornykiewicz, has carried out extensive pharmacologic trials on these two patients and has reported that study at this meeting.

I have referred to this subject because of the important neurophysiological and pharmacological implications and research challenge. The myoclonus has clinical features unlike the myoclonus of diffuse cerebral diseases and the pathology of this anoxic encephalopathy differs markedly from the usual one which is predominantly a laminar necrosis in cerebral cortex with appropriate cortical symptoms, and with seldom any but transient myoclonus. The pharmacological observations of this condition have proved important and rather exciting. Starting with Lhermitte, Peterfalvi, et al. in 1971 there have been a few drug trials and chemical tests which strongly suggest a serotonın transmitter disorder as a possible factor in this unique myoclonic syndrome produced by anoxic brain damage and presumably some focal neuronal depletion. Our two bring the total to nine reported patients who have been wholly, or almost wholly relieved of their myoclonus and ataxia by the precursor of serotonin, 5 HTP, L-5 hydroxytryptophan supported by carbidopa. This may prove to be an important new example of a motor mechanism disorder by a disease interfering with normal circuitry through depletion of a transmitter 
substance. Unlike L-dopa therapy for Parkinson's disease, this new treatment is directed only to a rare disease, but the stimulated research may well produce knowledge of major importance and applicability.

This commentary on problems of motor system disease as faced by the clinician has mentioned the complexity, sophistication and magnitude of modern research. The inevitable ultraspecialization of both neurologists and neuroscientists has been noted with a plea for retention, or perhaps restoration, of a certain number of highly skilled purely clinical neurologists. A review of the sources of knowledge of neurological disease and motor symptoms recalls a common pattern of alternating and mutually stimulating discoveries by the clinician and by the laboratory scientist. Wilson's disease and hypoxic action myoclonus exemplify such collaborative efforts and startling discoveries by study of disease manifestations and chemotherapy.

There remain many distressing quite unsolved diseases affecting motor mechanisms such as amyotrophic lateral sclerosis and dystonia musculorum. One hopes for a fortunate quick solution to these problems but the answers are more likely to come through circumscribed and prolonged fundamental research projects such as those to be presented at this Symposium.

\section{DISCUSSION}

Jasper felt that it was quite unusual that cases with this particular clinical picture resulting from anoxia would have only minimal damage to the cerebellum. Richardson emphasized that current views on the pathology of this disorder are based on a single post-mortem study by a group from France, who reported minimal loss of Purkinje cells but no other changes in the cerebellum. Richardson mentioned that one of the cases studied by him and Delean had recently died and that this case will be the subject of a detailed pathologic study. In view of the biochemical evidence for abnormalities in a serotoninergic system he felt that particular attention should be directed toward the raphe nuclei of the brain stem.

Zeldowicz (Vancouver) commented on some of the clinical similarities between these cases and the syndrome of Dysnergia Cerebellaris Myoclonica described by RamsayHunt and wondered whether the latter disorder might also respond to treatment with serotonin precursors.

Marshall (Ottawa) found it interesting that the cerebellar signs in one of Richardson's patients had been asymmetrical. He stated that the raphe nuclei are usually thought of as part of a diffuse monoaminergic system, one function of which is to control certain stages of sleep. That this system might also be capable of changing patterns of reflexes and resetting programs of motor activity was difficult to explain in the light of what is known about the anatomy of this diffuse system.

Richardson pointed out that the action myoclonus was fairly symmetrical and involved mainly trunk and proximal limb muscles, although the cerebellar intention tremor in his case was asymmetrical. Treatment with 5-hydroxytryptophan improved myoclonus, but intention tremor persisted.

\section{REFERENCES}

ARING, Charles D. (1965). A tribute to Sir Gordon Holmes. Journal of Nervous and Mental Disease, 5, 497-502.

CASTAIGNE, P., CAMBIER, J., ESCOUROLLE R., CATHALA, H. P. et LECASBLE, R. (1964). Observation anatomo-clinique d'un syndrome myoclonique post-anoxique. Revue Neurologique III, I, 60-73.

DENNY-BROWN, Derek (1965). A tribute to Sir Gordon Holmes. Journal of Nervous and Mental Disease, 5, 502-504.

HOLMES, Gordon (1939). The Cerebellum of Man. Brain, 62, 1-30.

LANCE, James W. and ADAMS, Raymond D. (1963). The Syndrome of Intention or Action Myoclonus as a Sequel to Hypoxic Encephalopathy. Brain, 86, 111-136.

LHERMitTe, F., PETERFAlvi, M., MARTEAU, R., GAZENGEL, J. et SERDARU, $M$. (1971). Analyse pharmacologique d'un cas de myoclonies d'intention et d'action postanoxiques. Revue Neurologique, Paris, 124, 21-31.

MANDELBROTE, B. M., STANIER, M. W., THOMPSON, R. H. S., THRUSTON, M. N. (1948). Studies on copper metabolism in demyelinating diseases of central nervous system. Brain, 71, 212-228.

STERNLIEB, I,, VAN DEN HORNER, C. J. A., MORELL, A. G., ALPERT, S., GREGORIADES, G. and SCHEINBERG, I. H. (1973). Lysosomal Defect of Copper Excretion in Wilson's Disease (Hepatolenticular Degeneration). Gastroenterology, 64, I, 99-105.

WALSHE, F. M. R. (1956). Preface. Selected Papers of Sir Gordon Holmes. VII-IX. MacMillan \& Co. Ltd., New York. 